\title{
Intracycle and Intercycle Interferences in Above-Threshold Ionization: the Time Grating
}

\author{
Diego G. Arbó ${ }^{1,2}$, Kenichi L. Ishikawa ${ }^{3}$, Klaus \\ Schiessl $^{4}$, Emil Persson ${ }^{4}$, and Joachim Burgdörfer ${ }^{4}$ \\ ${ }^{1}$ Institute for Astronomy and Space Physics, IAFE (Conicet-UBA), \\ CC 67, Suc. 28 (1428) Buenos Aires, Argentina \\ ${ }^{2}$ Department of Physics, FCEN, University of Buenos Aires, Argentina \\ ${ }^{3}$ Photon Science Center, Graduate School of Engineering, \\ the University of Tokyo, Hongo 7-3-1, \\ Bunkyo-ku, Tokyo 113-8656, Japan \\ ${ }^{4}$ Institute for Theoretical Physics, Vienna University of Technology, \\ Wiedner Hauptstraße 8-10/136, A-1040 Vienna, Austria, EU
}

(Dated: October 29, 2018)

\begin{abstract}
Within a semiclassical description of above-threshold ionization (ATI) we identify the interplay between intracycle and intercycle interferences. The former is imprinted as a modulation envelope on the discrete multiphoton peaks formed by the latter. This allows to unravel the complex interference pattern observed for the full solution of the time-dependent Schrödinger equation (TDSE) in terms of diffraction at a grating in the time domain. These modulations can be clearly seen in the dependence of the ATI spectra on the laser wavelength. Shifts in energy modulation result from the effect of the long Coulomb tail of the atomic potential.
\end{abstract}

PACS numbers: $32.80 . \mathrm{Rm}, 32.80 . \mathrm{Fb}, 03.65 . \mathrm{Sq}$ 
Tunneling ionization is a highly nonlinear quantum-mechanical phenomenon induced by intense laser pulses $\left(\gtrsim 10^{14} \mathrm{~W} / \mathrm{cm}^{2}\right)$. Electrons are emitted by tunneling through the potential barrier formed by the combination of the atomic potential and the external strong field. Tunneling has recently attracted increasing interest as a probe of the atomic and molecular structure [1, 2, 3]. Tunneling occurs within each optical cycle predominantly around the maxima of the absolute value of the electric field. The interference of the successive bursts of ejected electrons reaching the same final momentum gives rise to features in photoelectron energy and momentum distribution which are markedly different from typical above-threshold ionization (ATI) spectra by multi-cycle lasers. This temporal double-slit interference has recently been studied both experimentally [1, 4] and theoretically [5]. On the other hand, the ATI peaks separated by a photon energy can be themselves viewed as an interference pattern formed by electron bursts repeated each optical cycle. Details of the interplay between these intra- and intercycle interferences have not yet been clearly identified and analyzed, to the best of our knowledge.

In this Rapid Communication, we study the influence of different interference processes on ATI spectra generated by multi-cycle laser pulses. We clarify the underlying mechanism within a simple one-dimensional model employing classical trajectories. Within the framework of the strong-field approximation (SFA) [6] the qualitative features, the modulation of the ATI peaks akin to the modulation of Bragg peaks by the structure factor in crystal diffraction, can be unambiguously identified in the ATI spectrum determined from the full solution of the three-dimensional time-dependent Schrödinger equation (TDSE). The multi-cycle laser pulse thus acts as a diffractive grating in the time domain. Quantitative deviations between the SFA predictions and the full TDSE can be traced to the Coulomb tail of the atomic potential affecting this modulation. The latter opens up the opportunity to observe effects of the atomic potential in easy-to-obtain photoelectron spectra after ionization by multi-cycle laser pulses.

Our simple semiclassical model of photoelectron spectra is based on the 1D "simple man's model (SMM)" [6, 7, 8]. Let us consider an atom interacting with a linearly polarized laser pulse. The laser field $F(t)$ is chosen to be of the form $F(t)=f(t) \sin \omega t$, with an envelope function $f(t)$ corresponding to an $N$-cycle flat-top pulse with a field strength of $F_{0}$ and with $m$-cycle linear ramp-on and -off (we set $m=\frac{1}{2}$ in the following). The classical electron trajectories $i(i=1, \ldots, 2 N)$ for a final momentum $k$ are characterized by their release times 
$t_{r}^{(i)}$ which satisfy,

$$
k=-A\left(t_{r}^{(i)}\right),
$$

where $A(t)=-\int_{-\infty}^{t} d t^{\prime} F\left(t^{\prime}\right)$ denotes the vector potential divided by the speed of light, with $A(t)=\left(F_{0} / \omega\right) \cos \omega t$ for the flat-top segment of the pulse. In this study we focus on direct photoelectrons (without rescattering) with energies $E \lesssim 2 U_{p}\left(U_{p}=F_{0}^{2} / 4 \omega^{2}\right)$ which dominate the total ionization probability. It should be noted that within the flat-top part of the pulse and for a given value of $k$, the field strength upon ionization $\left|F\left(t_{r}^{(i)}\right)\right|$ is a constant independent of the release time $t_{r}^{i}(i=1, \ldots, 2 N)$. Thus, assuming that the ground-state depletion is negligible, the ionization rate $\Gamma\left(t_{r}^{(i)}\right)$ is identical for all the ionization bursts (or trajectories) to first approximation and only a function of $\Gamma(k)$. Consequently, the momentum distribution $P(k)$ can be written as

$$
P(k)=\Gamma(k)\left|\sum_{i=1}^{2 N} e^{i S\left(t_{r}^{(i)}\right)}\right|^{2},
$$

where $S$ denotes the Volkov action [9]

$$
S(t)=-\int_{t}^{\infty} d t^{\prime}\left[\frac{\left(k+A\left(t^{\prime}\right)\right)^{2}}{2}+I_{p}\right],
$$

with $I_{p}$ being the ionization potential. The key to the analysis of intracycle and intercycle interferences is that the sum over interfering trajectories (Eq. 2) can now be decomposed into those associated with two release times within the same cycle and those associated with release times in different cycles (see Fig. 1)

$$
\sum_{i=1}^{2 N} e^{i S\left(t_{r}^{i}\right)}=\sum_{j=1}^{N} \sum_{\alpha=1}^{2} e^{i S\left(t_{r}^{(j, \alpha)}\right)} .
$$

Accordingly, Eq. (2) becomes

$$
P(k)=\Gamma(k) \cos ^{2}\left(\frac{\Delta S}{2}\right)\left|\sum_{j=1}^{N} e^{i \bar{S}_{j}}\right|^{2}=\Gamma(k) F(k) B(k)
$$

where $\bar{S}_{j}=\left[S\left(t_{r}^{(j, 1)}\right)+S\left(t_{r}^{(j, 2)}\right)\right] / 2$ is the average action within each cycle and $\Delta S=S\left(t_{r}^{(j, 1)}\right)-$ $S\left(t_{r}^{(j, 2)}\right)$ is the difference. Note that $\Delta S$ is independent of $j$. Eq. (5) is structurally equivalent to the intensity for crystal diffraction: the factor $F(k)=\cos ^{2}(\Delta S / 2)$ represents the form factor (on structure) accounting for interference modulations due to the internal structure within the unit cell while the second factor $B(k)=\left|\sum_{j=1}^{N} e^{i \bar{S}_{j}}\right|^{2}$ gives rise to Bragg peaks 


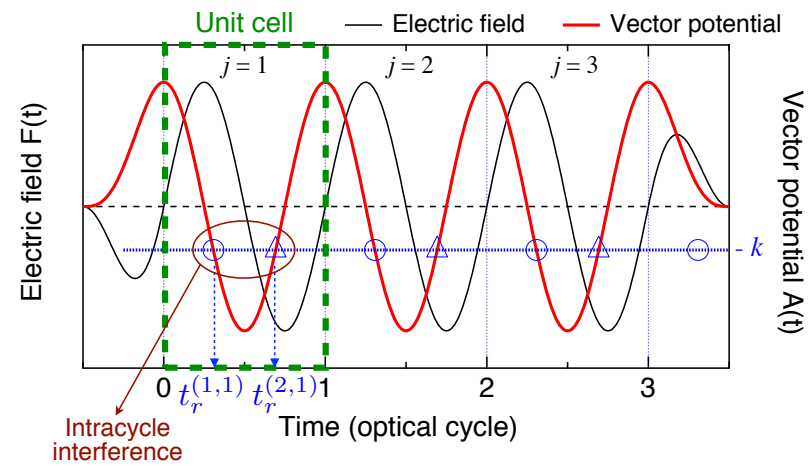

FIG. 1: (color online) Electric field $F(t)$ (left axis) and vector potential $A(t)$ (right axis) of a flat-top pulse $\left(N=4, m=\frac{1}{2}\right)$. The electron emission times for a given final momentum $k$ are marked by circles $\left(t_{r}^{(j, 1)}\right)$ and triangles $\left(t_{r}^{(j, 2)}\right)$. Each circle-triangle pair determines the structure factor $F(k)$ and leads to intracycle interference while the periodic train of such pairs gives rise to intercycle interference. Each optical cycle can be viewed as "unit cell" of the time lattice.

due to the periodicity of the crystals. Alternatively, Eq. (11) may be viewed as a diffraction grating in the time domain consisting of $N$ slits and with $F(k)$ being the diffraction factor for each slit. Interferences between different slits correspond to intercycle interferences while $F(k)$ represent intracycle interferences.

We show in the following that the interplay between $B(k)$ and $F(k)$ controls the direct ATI spectrum (Fig. 2). Note that the peak of the intracyle structure factor $F(k)$ are not equispaced in energy (shown in Fig. 2 (a) for hydrogen with $I_{P}=0.5$ a.u.). The separation of consecutive peaks is larger at intermediate energies than near the classical boundaries $E=0$ and $E=2 U_{p}\left(U_{p}=0.5\right.$ a.u. in the present case). It can be analytically shown that the separation between adjacent peaks $\Omega(k)$ as a function of the final momentum is given by

$$
\Omega(\kappa)=\frac{\omega \pi \kappa \sqrt{1-\kappa^{2}}}{1-\kappa^{2}+\gamma^{2} / 2-\kappa \sqrt{1-\kappa^{2}} \arccos \kappa},
$$

where $\gamma=\sqrt{2 I_{p}} \omega / F_{0}$ is the Keldysh parameter, and $\kappa=\omega k / F_{0}$ is the scaled dimensionless momentum. $\Omega(\kappa)$ reaches a maximum at $\kappa_{m}=\sqrt{1+\frac{\gamma^{2}}{2}-\frac{\gamma}{2} \sqrt{2+\gamma^{2}}}$, the position of which is indicated by a vertical arrow in Fig. 2 (a). Such intracycle interference patterns have been recently experimentally observed for ultrashort near-single cycle IR pulses [1, 4] and theoretically analyzed [5]. The intercycle interferences pattern (Fig. 2b) is, by contrast, equispaced and corresponds to the well-known ATI pattern with peak spacing corresponding 

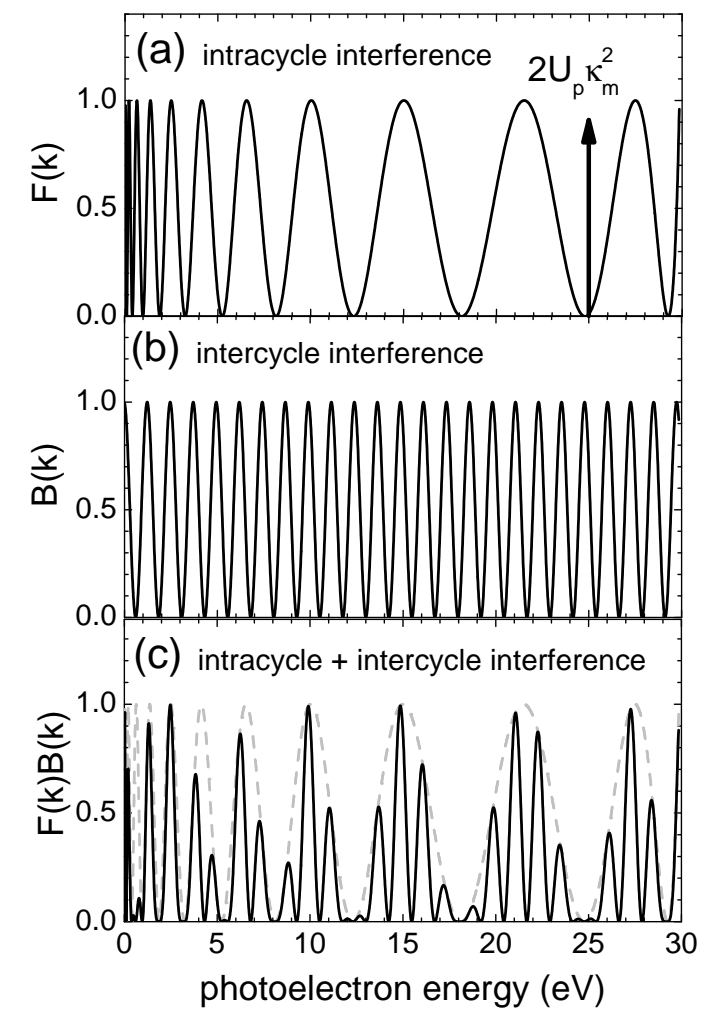

FIG. 2: Semiclassical intracycle and intercycle interference pattern (color online). (a) Intracycle interference pattern given by the structure factor $F(k)$. (b) Intercycle interferences with "Bragg" peaks given by $B(k)$ in Eq. (7). (c) Total semiclassical interference (Eq. (5) ) for $N=2$. The laser wavelength and intensity are $1000 \mathrm{~nm}$ and $1.6 \times 10^{14} \mathrm{~W} / \mathrm{cm}^{2}$, respectively. To stress the interferences, we set $\Gamma(k)=1$ and normalize the respective maxima of $F(k)$ and $B(k)$ to unity, see $\mathrm{Eq} \cdot(\underline{5})$.

to one-photon transitions $(\hbar \omega)$. For the case of $N=2$ in Fig. 2(b), $B(k)$ is given by

$$
B(k)=4 \cos ^{2}\left(\frac{S\left(t_{r}^{(1)}\right)-S\left(t_{r}^{(1)}+2 \pi / \omega\right)}{2}\right) .
$$

In multi-cycle photoelectron spectra with $N \geq 2$, both intracycle and intercycle interferences are simultaneously present. In Fig. 2 (c), the resulting energy distribution calculated from the time grating (Eq. (5) ) is shown for $N=2$. The multiphoton peaks are modulated by the intracycle interference structure factor. The ATI peaks become narrower as the number $N$ of optical cycles increases approaching, in this way, $\delta$-peaks for infinitely long 


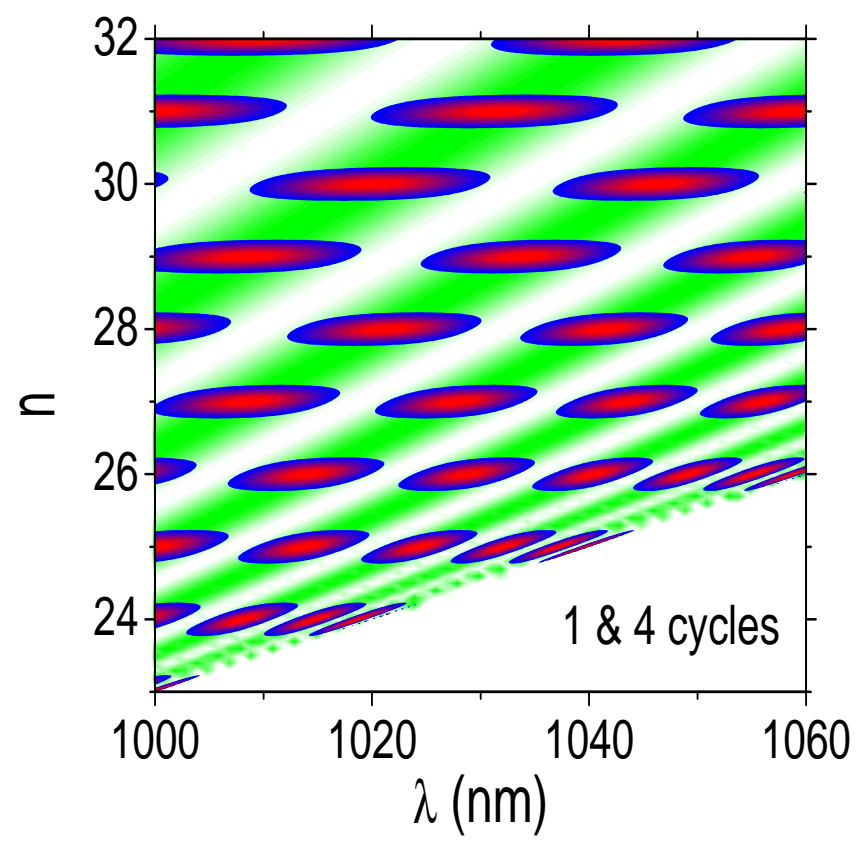

FIG. 3: (color online) Semiclassical total ionization probability as a function of the laser wavelength $\lambda$ and the ATI order $n$ (see text). Shown is the spectrogram for both a one-cycle pulse (green stripes) and and a four-cycle pulse (red islands). $F_{0}=0.0675$ a.u. $\left(1.6 \times 10^{14} \mathrm{~W} / \mathrm{cm}^{2}\right)$.

pulses. On the contrary, the intracycle modulation is independent of the number of cycles. Thus, the sub-cycle interference, previously studied with near-single-cycle ultrashort pulses, is embedded and visible in ATI spectra for multi-cycle pulses, a feature apparently up to now not fully recognized. This effect becomes more transparent when we study the parametric variation of the photoelectron spectrum. In Fig. 3 we show the spectrum expressed in units of the (new) photon number $n=\left(E+I_{p}+U_{p}\right) / \omega$, as a function of laser wavelength $\lambda$. For $N=4$ cycles the horizontal stripes in this two-dimensional interferogram peaking near 
integer values of $n$ represent ATI peaks due to intercycle interference. Superimposed are tilted stripes controlled by the intracycle interference, which are also visible for the $N=1$ cycle pulse. We note that a very similar two-dimensional interferogram has recently been found in atom-surface diffraction [10].

In view of the fact that the present intracycle and intercycle interference structures are derived from a simple one-dimensional SFA model neglecting realistic features of the atomic potential, we test its predictions against a full numerical 3D TDSE solution [11] for hydrogen. The resulting interferogram for photoelectrons (Fig. 4) agrees qualitatively remarkably well with the one-dimensional SFA model (Fig. 3). The intracycle modulation is best seen when the angular acceptance of the photoelectron spectrum is restricted to a cone of small angles around the polarization axis $\left(\theta=10^{\circ}\right.$ in Fig. 4a) while it is somewhat blurred but still visible in the total spectrum (Fig. 4b). We found similar interference patterns for rare gases such as argon, though the details depend on the atomic potential. Projecting the two-dimensional distribution onto the $\lambda$ axis (Fig. 4c) results in a regular modulation pattern on a fine $\lambda$ scale which can be traced back to the combined effect of inter- and intracycle interferences. This oscillations closely resemble those previously observed for the $\lambda$ dependence of HHG [12, 13, 14].

Experimentally, more easily accessible is the variation of the photoelectron spectra as a function of the energy at fixed wavelength, i.e., projection of the $2 \mathrm{D}$ interferogram onto the $\lambda$ axis (Fig. 5). The photoelectron spectrum clearly reflects the modulation of the regularly spaced ATI peaks (intercycle interference) by intracycle interference, in complete agreement with the prediction of a time grating (Eq. (5). It is now highly instructive to probe this interference pattern for its dependence on the Coulomb potential neglected in the SFA derivation of the time grating. Within the full TDSE we can explore Coulomb-tail effects by using a screened Coulomb potential,

$$
V(r)=\left\{\begin{array}{ll}
-\frac{1}{r} & \left(r<r_{c}\right) \\
-\frac{e^{-\left(r-r_{c}\right) / r_{d}}}{r} & \left(r>r_{c}\right)
\end{array},\right.
$$

where the parameter $r_{d}$ characterizes the width of the transition from the full to the screened Coulomb potential. We use $r_{d}=10$ a.u., and $r_{c}$ is varied between 10 and 70 a.u. For these parameter values, the ionization potential and the first excitation energy remain unchanged to an accuracy ranging between $10^{-3}$ and $10^{-9}$. Consequently, the position of the ATI peaks remains unchanged as a function of $r_{c}$. However, the modulation envelope is systematically 


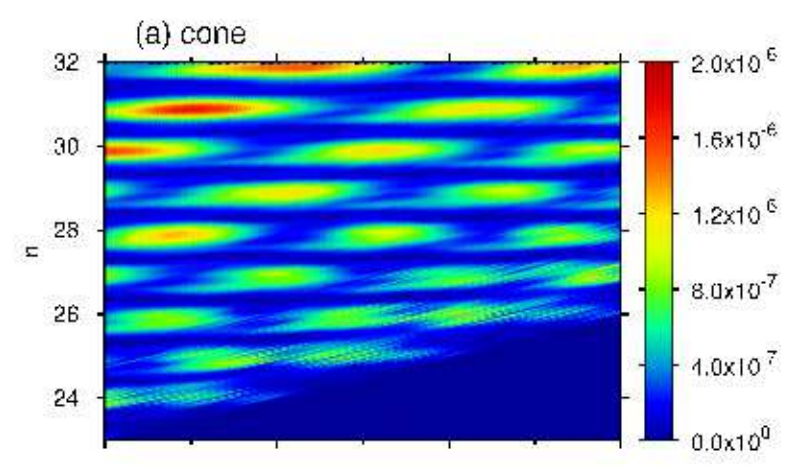

(b) all angles
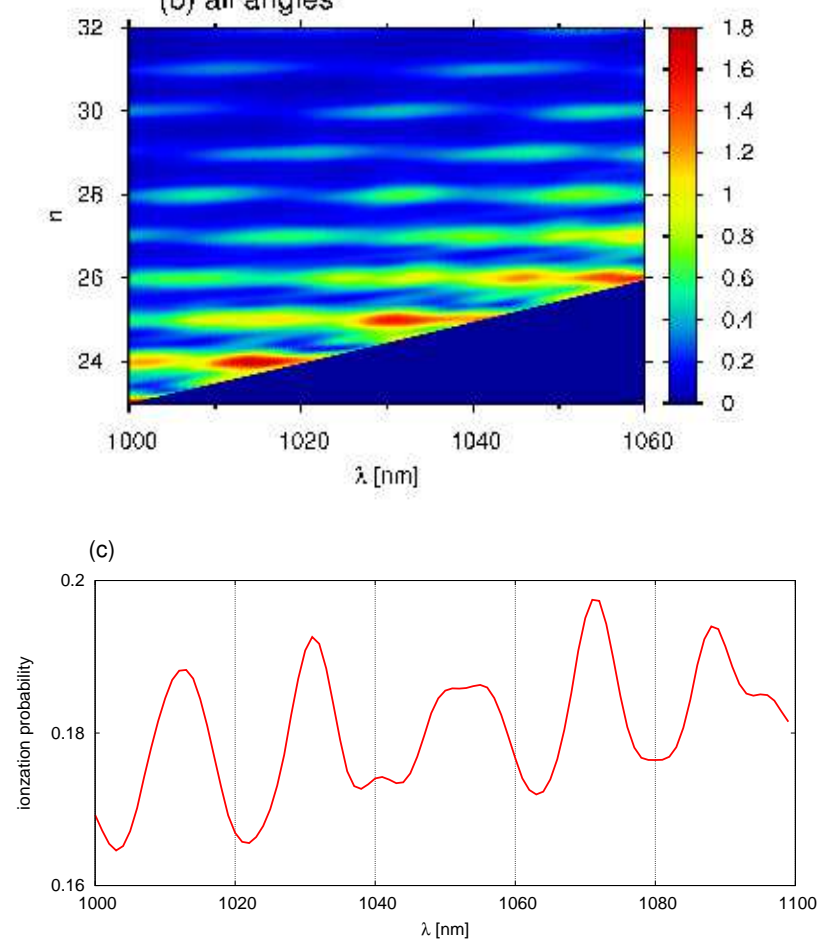

FIG. 4: (color online) Two-dimensional interferogram as a function of laser wavelength $\lambda$ and ATI order $n$ (see text) calculated by using the TDSE for hydrogen for a four-cycle pulse with $F_{0}=0.0675$ a.u. $\left(1.6 \times 10^{14} \mathrm{~W} / \mathrm{cm}^{2}\right)$. (a) Emission into a cone of $10^{\circ}$ around the polarization axis and (b) for all angles. (c) Energy-integrated total ionization yield as a function of $\lambda$.

and almost rigidly shifted towards lower energies under the influence of the long-range Coulomb tail. The Coulomb phase does not affect the Bragg peaks but changes the structure factor $F(k)$ of the time grating in Eq. (5) . Thus, the Coulomb potential is responsible for a shift in the positions of the intracycle interference stripes, visible in Figs. 3 and 4(a). It should be emphasized that the shift is equally significant even for higher photoelectron 


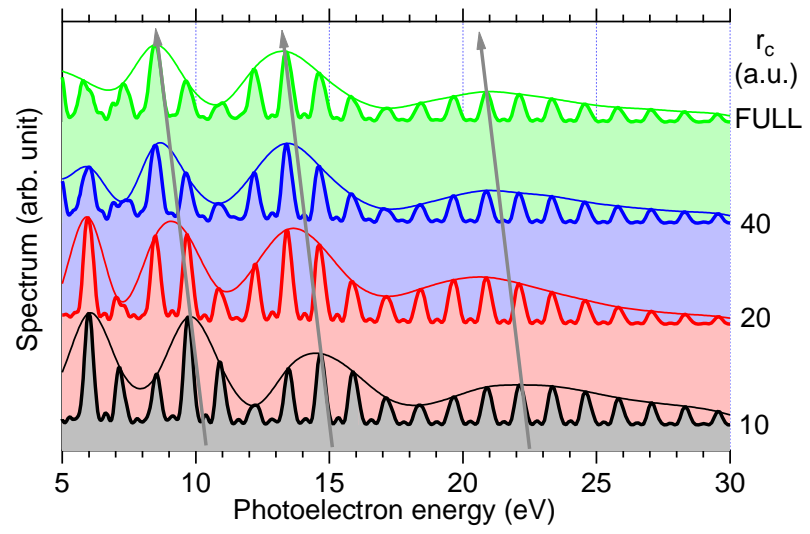

FIG. 5: (color online) Comparison of the photoelectron spectra within the $10^{\circ}$ cone, calculated with the full Coulomb potential (marked as "full") with the truncated Coulomb potentials for varying values of $r_{c}$ as indicated. The laser wavelength is $1000 \mathrm{~nm}$. Other laser parameters are the same as in Fig. 4. Thin solid lines are approximate peak envelopes.

energies, for which one might expect the SFA to be better justified. We note parenthetically that such shifts can be partially accounted for by including the Coulomb-Volkov phase into the semiclassical theory [14, 15].

In summary, we have identified the interplay between the intra- and intercycle interferences of electron trajectories in ATI spectra by intense multicycle laser pulses. The former, which carries information on attosecond subcycle dynamics of the electron cloud [1], is imprinted as a pulse-length independent modulation of multiphoton peaks formed by the latter. This modulation is even more clearly visible in the wavelength dependence of ATI spectra, which could be measured with state-of-the-art tunable sources based, e.g., on optical parametric chirped pulse amplification. While the general features of the interplay between the intracycle and intercycle interferences is well explained by the simple semiclassical theory, the intracycle modulation envelope is shifted by the Coulomb tail which emphasizes the effect from the atomic potential.

This work was supported by CONICET and PICT2006-00772 of ANPCyT (Argentina), SFB 016 ADLIS and P15025-N08 of FWF (Austria), and by the EU project HPRI-200150036. K.L.I. gratefully acknowledges financial support by the JST-PREST program, the MEXT Grant No. 19686006 (Japan), and the Advanced Photon Science Alliance (APSA) project (Japan) and KS acknowledges support by the IMPRS program of the MPQ (Germany) 
[1] R. Gopal et al., Phys. Rev. Lett. 103, 053001 (2009).

[2] M. Meckel, D. Comtois, D. Zeidler, A. Staudte, D. Pavicic, H. C. Bandulet, H. Pépin, J. C. Kieffer, R. Dörner, D. M. Villeneuve, and P. B. Corkum, Science 320, 1478 (2008).

[3] D. G. Arbó, S. Yoshida, E. Persson, K. I. Dimitriou, and J. Burgdörfer, Phys. Rev. Lett. 96, $143003(2006)$.

[4] F. Lindner, M. G. Schätzel, H. Walther, A. Baltuška, E. Goulielmakis, F. Krausz, D. B. Milošević, D. Bauer, W. Becker, and G. G. Paulus, Phys. Rev. Lett. 95, 040401 (2005).

[5] D. G. Arbó, E. Persson, and J. Burgdörfer, Phys. Rev. A 74, 063407 (2006); D. G. Arbó, S. Yoshida, E. Persson, K. I. Dimitriou, and J. Burgdörfer, Journal of Physics: Conferences Series 88, 012054 (2007).

[6] M. Lewenstein, K. C. Kulander, K. J. Schafer, and P.H. Bucksbaum, Phys. Rev. A 51, 1495 (1995); M. Lewenstein, Ph. Balcou, M. Yu. Ivanov, A. L'Huillier, and P. B. Corkum, Phys. Rev. A 49, 2117 (1994).

[7] N. B. Delone and V. P. Krainov, J. Opt. Soc. Am. B 8, 1207 (1991).

[8] C. C. Chirila and R. M. Potvliege, Phys. Rev. A 71, 021402(R) (2005).

[9] D. M. Volkov, Z. Phys. 94, 250 (1935).

[10] F. Aigner et al., private communication.

[11] X.-M. Tong and S. Chu, Chem. Phys. 217, 119 (1997).

[12] K. Schiessl, K. L. Ishikawa, E. Persson, and J. Burgdörfer, Phys. Rev. Lett. 99, 253903 (2007).

[13] K. Schiessl, K. L. Ishikawa, E. Persson, and J. Burgdörfer, Jour. Mod. Opt. 55, 2617 (2008).

[14] K. L. Ishikawa, K. Schiessl, E. Persson, and J. Burgdörfer, Phys. Rev. A 79, 033411 (2009).

[15] D. G. Arbó, J. E. Miraglia, M. S. Gravielle, K. Schiessl, E. Persson, and J. Burgdörfer. Phys. Rev. A, 77, 013401, 2008. 